\title{
Nanotechnology and the promise for enhanced cancer chemotherapy
}

\author{
Anthony J. Apostoli
}

Queen's University

Cancer chemotherapy has evolved since the serendipitous discovery in the 1940s that mustard gas compounds could stop the uncontrolled proliferation of white blood cells in lymphoma patients ${ }^{1}$. More effective whole-body chemotherapeutic agents aimed at a broad range of cancers have been developed since then; however, their success is limited by severe toxicity to normal cells ${ }^{2}$. Recently, targeted chemotherapies have emerged, specifically targeting cancer cell components with fewer side effects ${ }^{3}$. Nevertheless, ongoing issues with identifying cancer cell-specific targets, determining which patients will respond to a particular targeted therapy and the development of chemoresistance limit the effectiveness of these drugs in humans. To this end, scientists have begun to harness the potential of nanotechnology - a scientific realm that focuses on manipulating matter into cell-sized instruments - to overcome some of these issues and improve efficacy of current cancer chemotherapy ${ }^{4}$.

A major challenge today in cancer drug development is target cell specificity. Current anti-cancer agents are administered systemically per oral or intravenous routes and diffuse throughout the body where they interact with both cancerous and normal cells, and are actively metabolized by the liver and kidneys. These actions contribute to a reduction in the therapeutic index, safety, specificity, and bioavailability of drugs $^{5}$ that may be overcome by nanotechnological approaches. Nanoparticles are polymeric or inorganic structures (ranging from $1-500 \mathrm{~nm}$ in size) designed to carry and deliver highly concentrated anti-cancer compounds specifically to tumour sites. There are two ways in which this is carried out. The passive targeting strategy exploits the inherent "leakiness" and poor drainage of cancerous tissues ${ }^{6}$. Smaller nanoparticles tailored for this strategy can selectively accumulate within the tumour environment to release drug cargo. In contrast, the active targeting strategy involves conjugating target ligands, which are specific to membrane receptors overexpressed on tumour cells, to the surface of the nanoparticle structure. These ligands facilitate the interaction between the nanoparticle and the tumour cell, and trigger receptor-mediated endocytosis for subsequent delivery of the payload directly into the cancer cell ${ }^{7,8}$.

The potential for targeted drug delivery via nanoparticles has important implications for the refinement of cancer chemotherapeutics. Researchers have begun to experiment with more traditional whole-body chemotherapies, whose side effects may have limited their use and effective dosage. In the pre-clinical setting, some of these agents have been encapsulated in nanoparticles and delivered to tumours at safe and effective doses ${ }^{9,10}$. When delivered in this manner, the therapeutic benefit of these anti-cancer drugs generally outweighed the observed side effects ${ }^{4}$. This suggests that more research is needed to evaluate the efficacy of other traditional whole-body chemotherapies deemed too risky for human use.

The promise of nanotechnology requires that more cancer cell-specific targets and phenotypes are identified that can be exploited by these drug delivery vectors to treat various types of cancers. This is a difficult task given the fact that cancer cells hijack normal cells. Simply employing the active targeting strategy aimed at blatant tumour specific properties may also elicit adverse effects on normal cells which share these properties. For example, an obvious target in breast cancer is human epidermal growth factor receptor 2 (HER2), which is known to be amplified in $\sim 30 \%$ of tumours ${ }^{11}$. While this protein is also expressed on the surface of normal cells, albeit to a lesser extent, actively targeting HER2 may contribute to normal cell toxicity. One way to potentially minimize these side effects is through the 
identification of other receptors found specifically on HER2-overexpressing tumours. Gene expression profiling of a cohort of HER2-positive breast cancers may identify several putative targets which could collectively be loaded to the nanoparticle surface to ensure more exclusive drug delivery to these cancer cells. A similar strategy could be employed to identify multiple potential targets specific to triple-negative breast cancer, which is one of the most difficult subtypes to treat.

In the future, it would be imperative to test the design of a nanoparticle incorporating both the active and passive targeting strategies. This could be achieved by altering the immediate physical structure of the drug-transport vector for passive targeting, as well as refining the biochemical ligand-receptor binding properties to better identify the cancer target for active targeting. In principle, the passive strategy would ensure that most nanoparticles remain in the vicinity of the tumour environment limiting the effects of active targeting to normal cells in a particular site of the body.

Many particle-based drug delivery systems are currently being assessed in clinical trials, yet only a few have been approved and marketed for human use. This may be due to the scantily available toxicological data for these systems or the high costs associated with large-scale production ${ }^{12}$. With the nanotechnological realm in rapid expansion, there is still much to be explored before new cancer treatment modalities will become clinically available. Nonetheless, the future looks promising for cancer patients, biologists and drug developers alike.

\section{Acknowledgements}

I would like to thank Dr. Christopher Nicol in the Department of Pathology \& Molecular Medicine, as well as Diana Marsilio, Graham Skelhorne-Gross, and Ted Nagaria for their valuable feedback during the preparation of this article.

\section{References}

1. DeVita VT, Jr., Chu E. A history of cancer chemotherapy. Cancer Res. 2008;68:8643-8653.

2. Leyland-Jones B, Semiglazov V, Pawlicki M, et al. Maintaining normal hemoglobin levels with epoetin alfa in mainly nonanemic patients with metastatic breast cancer receiving first-line chemotherapy: a survival study. J Clin Oncol. 2005;23:5960-5972.

3. Schally AV, Nagy A. Cancer chemotherapy based on targeting of cytotoxic peptide conjugates to their receptors on tumors. Eur J Endocrinol. 1999;141:1-14.
4. Liu Y, Solomon M, Achilefu S. Perspectives and potential applications of nanomedicine in breast and prostate cancer. Med Res Rev. 2010.

5. Kaufmann SH, Earnshaw WC. Induction of apoptosis by cancer chemotherapy. Exp Cell Res. 2000;256:42-49.

6. Iyer AK, Khaled G, Fang J, Maeda H. Exploiting the enhanced permeability and retention effect for tumor targeting. Drug Discov Today. 2006;11:812-818.

7. Qian ZM, Li H, Sun H, Ho K. Targeted drug delivery via the transferrin receptor-mediated endocytosis pathway. Pharmacol Rev. 2002;54:561-587.

8. Sun C, Sze R, Zhang M. Folic acid-PEG conjugated superparamagnetic nanoparticles for targeted cellular uptake and detection by MRI. J Biomed Mater Res A. 2006;78:550-557.

9. Lau KG, Hattori Y, Chopra S, et al. Ultra-deformable liposomes containing bleomycin: in vitro stability and toxicity on human cutaneous keratinocyte cell lines. Int J Pharm. 2005;300:4-12.

10. Zhang Y, Zhuo RX. Synthesis and drug release behavior of poly (trimethylene carbonate)-poly (ethylene glycol)poly (trimethylene carbonate) nanoparticles. Biomaterials. 2005;26:2089-2094.

11. Vivanco I, Mellinghoff IK. Epidermal growth factor receptor inhibitors in oncology. Curr Opin Oncol. 2010;22:573-578.

12. Solomon M, D'Souza G G. Recent progress in the therapeutic applications of nanotechnology. Curr Opin Pediatr. 2011;23:215-220.

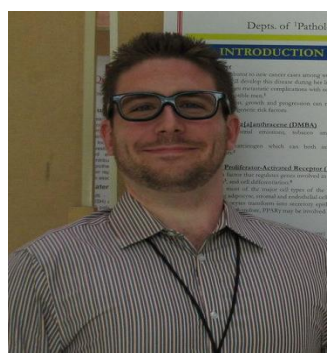

Author Profile

Anthony Apostoli is a current $\mathrm{PhD}$ candidate at Queen's University in Kingston, Ontario. His research interests include the stem cell theory of cancer and breast tumour biology. He enjoys long walks on the beach, camping with his fiancée, and western blotting. 